Editorial

\title{
Biomarkers for cancer immunotherapy... status in the clinic
}

\section{Introduction}

To initiate this presentation, we should briefly describe the role of the immune system in cancer, this is termed immune surveillance.

There are three stages in the mechanism of immune surveillance: elimination, equilibrium and escape. First, cells undergoing malignant transformation are recognized and eliminated by the immune system because of their high intrinsic immunogenicity. Follows, a stage which is a period of equilibrium between cancer cell growth and the immune response. In the third stage, through processes such as immunoediting and expression of co-inhibitory ligands (checkpoint proteins), cancer cells find a way to escape immune surveillance and grow into clinically overt disease.

\section{Immunotherapy platforms}

Some cancer immunotherapies target specific steps in the cancerimmune system interaction cycle. Of particular interest to this presentation are the immune checkpoint inhibitors. The immune system depends on multiple checkpoints to avoid overactivation that could damage and kill healthy cells. Tumor cells often take advantage of these immune checkpoints to escape detection by the immune system. CTLA-4 and PD-1/PD-L1 are immune checkpoints that have been determined as targets for cancer therapy.

CTLA-4 is upregulated on the surface of T cells when they recognize novel cancer antigens presented on the surface of dendritic cells. CTLA-4 inhibitors work at theT-cell priming and activation step of the cancer-immune system interaction cycle. PD-1 is another immunologic checkpoint that has been found to be upregulated in a variety of tumors; PD-1 and one of its known ligands, PD-L1, are involved in the final step of the interaction between activated $\mathrm{T}$ cells and tumor cells. The binding of PD-1 to PD-L1 prevents the activated T cell from killing tumor cells. Inhibitors to PD-1 and PD-L1 prevent this binding, allowing the $\mathrm{T}$ cell to release factors such as perforin that kill the target tumor cells via the process of apoptosis. Checkpoint inhibitors that target PD-1 and PD-L1 showed activity in several different tumor types, particularly melanoma, lung cancer and kidney cancer.

Three immune checkpoint inhibitors are currently approved for specific indications in patients with cancer: Nivolumab, Pembrolizumab and Ipilimumab.

\section{PD-LI, a biomarker for immune checkpoint inhibitors}

As is true with other agents targeting specific molecular mechanisms, there are advantages to identifying patients who are the most likely to benefit from treatment with immune checkpoint inhibitors.

Multiple studies across a diversity of tumor types have identified a correlation between the pretreatment expression level of PD-L1 in the tumor microenvironment and the likelihood of response to
Volume 4 Issue 4 - 2016

Hajj Adel Anis

Cedars - Jebel Ali International Hospital, UAE

Correspondence: Haji Adel Anis, Medical Oncologist at Cedars - Jebel Ali International Hospital, 9370 Rue Lajeunesse, Montreal, UAE, Tel 438-992-55I6, Email ahajj@dr.com

Received: February 06, 2016 | Published: March 03, 2016 single-agent PD-1 pathway inhibitor therapy. However, many of the current assays are proprietary and are being developed as companion diagnostic assays for specific immune checkpoint inhibitors. These assays use different antibodies, different staining protocols, different target cell assessment (tumor cells, tumor-infiltrating immune cells, or both), different scoring methods (percentage of staining cells, IHC 1-3) and different thresholds for defining a positive test result. Currently, there is no such thing as a defined, definite PD-L1 positive result across assays like we think about for BRAF and EGFR mutation testing for example, therefore, testing for PD-L1 expression is most useful for stratifying patients for clinical trials using PD-1 pathway inhibitors.

\section{PD-LI IHC assays for clinical decision making}

At this point in time, it appears that we are not ready to use PDL1 IHC assays to select patients for PD-1 pathway inhibitor therapy; even the prognostic value of PD-L1 expression is questionable. PDL1 expression has been associated with poor prognosis in patients with kidney cancer, but good prognosis in patients with melanoma. As yet, we do not understand this distinction. The use of PD-L1 IHC assays will depend rather, on the type of cancer; it is not a "one-sizefits-all" approach.

In the CheckMate 067 trial that compared the combination of nivolumab plus ipilimumab vs single-agent nivolumab vs singleagent ipilimumab as first-line therapy for patients with advanced melanoma, the patients identified as PD-L1 negative appeared to show better outcomes with the combination regimen than with either single agent. Based on these results, testing patients with advanced melanoma for PD-L1 expression to guide the choice of treatment would not be relevant since it looks not able to drive to a conclusive preference for a specific agent.

On the other side, it has been shown in several trials that for NSCLC, there is clearly a relationship between testing positive for PD-L1 expression and the likelihood of response to PD-1 pathway inhibitors. Whether this translates into an impact on survival is a different question. For patients with nonsquamous cell NSCLC, PD-L1 expression did appear to predict for survival benefit with nivolumab. There was a significant difference in response rates for 
patients who had no PD-L1 expression vs at least minimal PD-L1 expression, in favor of patients with higher thresholds to define positive PD-L1 expression. On the contrary, for most patients in the second-line setting of advanced squamous cell NSCLC we would not use a PD-L1 IHC assay to identify patients who should or should not be treated with a PD-1 pathway inhibitor; a survival benefit was seen in patients with PD-L1 positive and PD-L1 negative tumors. So, in brief, a PD-L1 IHC assay is not useful for patients with squamous cell NSCLC but may be useful for patients with nonsquamous cell NSCLC.

It would be justifiable to suggest that one reason the PD-L1 IHC assays are less relevant for patients with melanoma is because these tumors are much more immunogenic, much more mutated tumors than other types of tumors. So even if melanomas are negative for PDL1 expression, they are most likely still very immunogenic. On the other hand, lung cancer and many other types of tumors may have a much broader range of genetic damage. Eventually we will be brought to use a range of information including tumor mutational profile, the presence of infiltrating T cells and PD-L1 expression level, to make clinical decisions about treatment. PD-L1 expression assessment will be only one aspect of that information.

\section{Conclusion}

Currently, testing for PD-L1 expression is most useful for stratifying patients for clinical trials using PD-1 pathway inhibitors and potentially for determining which diseases to treat. PD-L1 expression in the tumor microenvironment has been shown to enrich for response to single agent PD-1/PD-L1 checkpoint blockade in a number of different tumor types. However, this relationship remains investigational and should not be used to select patients who should or should not be treated with these agents. PD-L1 expression does not appear to be useful for assessing response to anti-PD-1 plus antiCTLA-4 therapy in patients with metastatic melanoma.

By getting a better multifactorial assessment of what is occurring within the tumor and its microenvironment, including biomarker assays-PD-L1 expression, we should be better able to rationally deliver personalized therapy, mono or combination immunotherapy. 